\title{
Extra Ordinary Properties of Titanium Useful in Various Sectors and Enlightenment of "The Kroll Process"
}

\author{
Mali Bhushan Ishwar* \\ Department of Mechanical Engineering, Savitribai Phule Pune University, India \\ Accepted 02 March 2016, Available online 15 March 2016, Special Issue-4 (March 2016)
}

\begin{abstract}
Because of light weight, high strength to weight ratio, low modulus of elasticity, and excellent corrosion resistance, titanium and some of its alloys having important properties for the aerospace industry since the 1950s. Now, with the additional advantages of excellent biocompatibility, good local spot weld ability, and easy shaping and finishing by a number of mechanical and electrochemical processes, these materials are finding uses in dental applications, such as implants and restorative castings. Although more research is still needed in areas such as development of optimal casting investments, porcelain veneering systems, device designs, and controlled biological responses, the present and future uses of titanium appear bright for human implant.
\end{abstract}

Keywords: Titanium, Strength, Biocompatibility, Implants, Dentistry.

\section{Introduction}

Titanium is known as a transition metal on the periodic table of elements denoted by the symbol "Ti". It has silver gray color and it is light weight material. It's atomic number is 22 and an atomic weight of 47.90 . It has density of $4510 \mathrm{~kg} / \mathrm{m}$, which has somewhere between the densities of aluminium and stainless steel. It has melting point $1943.15 \mathrm{~K}$ and a boiling point is about $3560.15 \mathrm{~K}$. It behaves chemically similar to zirconium and silicon. It has awesome corrosion resistance and high strength.

Titanium rarely found in its pure form, titanium typically exists in minerals such as anatase, brookite, ilmenite, leucoxene, perovskite, rutile, and sphene. While titanium is relatively abundant, it continues to be expensive because it is difficult to isolate. The leading producers of titanium concentrates include Australia, Canada, China, India, Norway, South Africa, and Ukraine. In the United States, the primary titanium producing states are Florida, Idaho, New Jersey, New York, and Virginia.

Thousands of titanium alloys have been developed and these can be grouped into four main categories. Their properties depend on their basic chemical structure and the way they are manipulated during manufacture. Some elements used for making alloys

*Corresponding author: Mali Bhushan Ishwar include aluminium, molybdenum, cobalt, zirconium, tin, and vanadium. Alpha phase alloys have the lowest strength but are formable and wieldable. Alpha plus beta alloys have high strength. Near alpha alloys have medium strength but have good creep resistance. Beta phase alloys have the highest strength of any titanium alloys but they also lack ductility.

\section{Titanium's History}

Titanium is discovered by William gregor at 1791 in England. The origin name of this material is 'titan' means the sons of the earth goddess in greek mythology. Several years later, the same element was rediscovered by german chemist, klaproth.

Both klaproth and gregor worked with titanium compounds. Isolation of the pure metal was not demonstrated until 1910.This process produced individual pieces of pure titanium. These demonstrations prompted William Kroll to begin experimenting with different methods for efficiently isolating titanium. These early experiments led to the development of a process for isolating titanium by reduction with magnesium in 1937. This process, now called the Kroll process, is still the primary process for producing titanium. The first products made from titanium were introduced around the 1940s and included such things as wires, sheets, and rods. 


\subsection{Historical Utilization of Titanium}

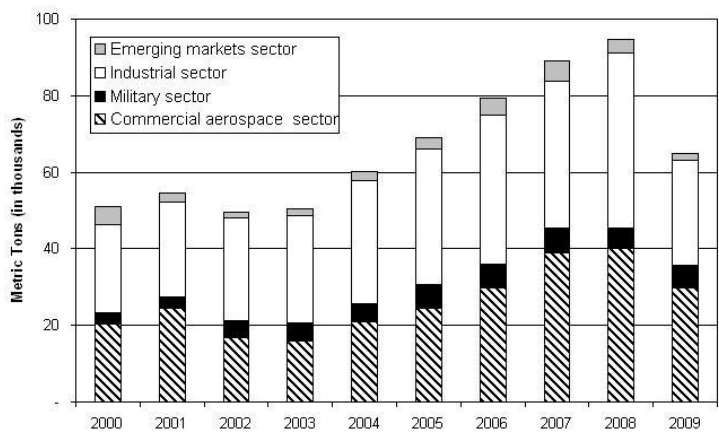

Chart.1 Historical Utilization of Titanium

\section{About Titanium}

\subsection{Structure of Titanium}

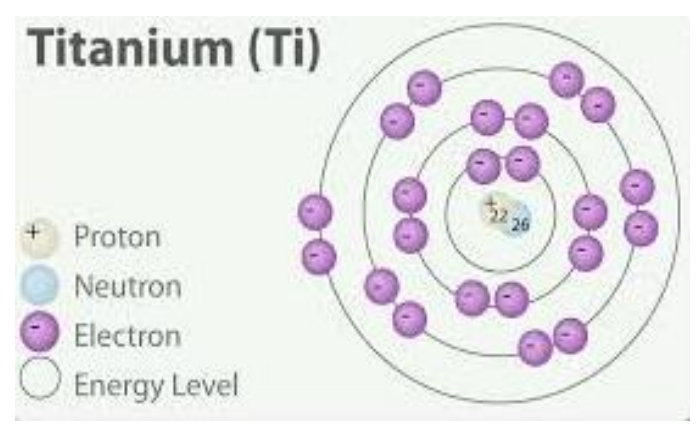

Fig.1 Structure of Titanium

\subsection{Fact Box}

Table.1 Initial Parameters

\begin{tabular}{|c|c|c|}
\hline $\begin{array}{r}\text { Sr. } \\
\text { No. }\end{array}$ & Parameters & Values \\
\hline 1 & Atomic Number & 22 \\
\hline 2 & Atomic Mass Number & 40 \\
\hline 3 & State at Room Temp. & Solid \\
\hline 4 & Melting Point & $1943.15 \mathrm{~K}$ \\
\hline 5 & Boiling Point & $3560.15 \mathrm{~K}$ \\
\hline 6 & Density & $4508 \mathrm{~kg} \mathrm{~m}^{-3}$ \\
\hline 7 & Relative Atomic Mass & 47.867 \\
\hline
\end{tabular}

\section{Occurrence of Titanium in Nature}

Titanium is always bonded to other elements in nature. It is widely distributed and occurs primarily in the minerals anatase, brookite, ilmenite, perovskite, rutile and titanite (sphene).Of these minerals, only rutile and ilmenite have economic importance, yet even they are difficult to find in high concentrations.

Significant titanium exist in western Australia, Canada, China, India, Mozambique, New Zealand, Norway, Ukraine and South Africa. About 186,000 tons of titanium metal sponge were produced in 2011, mostly in China $(60,000$ t), Japan $(56,000$ t), Russia $(40,000 \mathrm{t})$, United States $(32,000)$.
Titanium is contained in meteorites and has been detected in the sun and in M-type stars, which are the coolest type of star, with a surface temperature of $3,473 \mathrm{~K}$. Rocks brought back from the moon during the Apollo 17 mission are composed of $12.1 \% \mathrm{TiO}_{2}$. It is also found in coal ash, plants, and even in the human body.

\section{Manufacturing of Titanium by "Kroll Process"}

Titanium is produced using the Kroll process. The steps involved include extraction, purification, sponge production, alloy creation.

\subsection{Extraction}

At the start of production, the manufacturer receives titanium concentrates from mines. These materials are put in a fluidized-bed reactor along with chlorine gas and carbon.[4] The subsequent chemical reaction results in the creation of impure titanium tetrachloride (TiCl4) and carbon monoxide. Therefore the various unwanted metal chlorides that are produced must be removed.

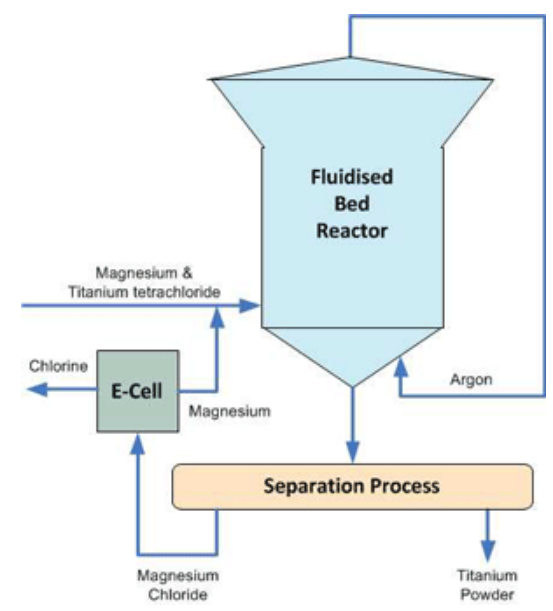

Fig.2 Separation by fluidized bed reactor

\subsection{Purification}

The reacted metal is put into large distillation tanks and heated. During this step, the impurities are separated using fractional distillation and precipitation. This action removes metal chlorides including those of iron, vanadium, zirconium, silicon, and magnesium.

\subsection{Production of sponge}

Next, the purified titanium tetrachloride is transferred as a liquid to a stainless steel reactor vessel. Magnesium is then added and the container is heated to about $1,373 \mathrm{~K}$. Argon is pumped into the container so that air will be removed and contamination with oxygen or nitrogen is prevented. The magnesium reacts with the chlorine producing liquid magnesium 
chloride. This leaves pure titanium solid since the melting point of titanium is higher than that of the reaction.

The titanium solid is removed from the reactor by boring and then treated with water and hydrochloric acid to remove excess magnesium and magnesium chloride. The resulting solid is a porous metal called a sponge.

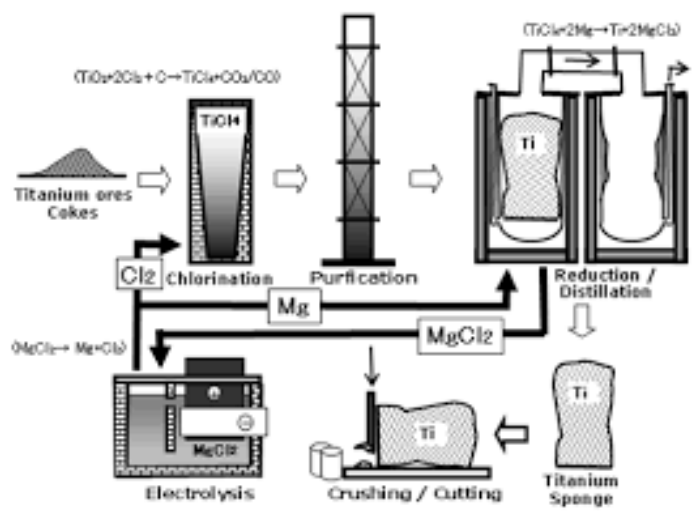

Fig.2 Sponge production

\subsection{Alloy creation}

The pure titanium sponge can then be converted into a usable alloy via a consumable-electrode arc furnace. At this point, the sponge is mixed with the various alloy additions and scrap metal. The exact proportion of sponge to alloy material is formulated in a lab prior to production. This mass is then pressed into compacts and welded together, forming a sponge electrode.

\section{Physical Specialties}

Titanium material is also recognized for high strength to weight ratio. It is very strong metal to low density that is quite ductile[3]. And it is lustrous material with metallic white in color. It has relatively high melting point $1943.15 \mathrm{~K}^{\circ}$ makes it useful as a refractory metal. It has low conductivity to electricity and thermal also. Commercial grade of titanium has ultimate tensile strength of about $434 \mathrm{Mpa}$, equal to that of common, low grade steel alloys, but are less dense. Titanium is $60 \%$ more dense than aluminium, but more than twice as strongas the most commonly used aluminium alloy. Certain titanium alloys achieve tensile strengths of over $1400 \mathrm{MPa}$.

Titanium is fairly hard (although not as hard as some grades of heat-treated steel), non-magnetic and a poor conductor of heat and electricity. Machining requires precautions, as the material will soften and gall. If sharp tools and proper cooling methods are not used. Like those made from steel, titanium structures have a fatigue limit which guarantees longevity in some applications loses strength when heated above $430{ }^{\circ} \mathrm{C}$. Titanium alloys have lower specific stiffness than in many other structural materials such as aluminium alloys and carbon fiber.
Titanium is as strong as steel, yet $45 \%$ lighter. Titanium alloy will work continuously at temperatures up to $600^{\circ} \mathrm{C}$.

\section{Conclusions}

- $\quad$ Titanium has very high strength to weight ratio hence is widely used in aerospace sector and also in armor field.

- Because of light weight, high strength to weight ratio, low modulus of elasticity, and excellent corrosion resistance, titanium and some of its alloys have been important properties for the aerospace industry.

- This material is widely use in aircraft jet engines, Industrial gas turbines, Automotive industry, Computer industry due to its corrosion resistance.

- It also has low density than other materials but the hardness is more than Aluminium Alloy.

- It has low thermal and poor electrical conductivity hence it can use in most safety operations.

- Machining requires precautions, as the material will soften and gall. It is mandatory to use sharp tools and proper cooling medium.

- Titanium alloys have lower specific stiffness than in many other structural materials such as aluminium alloys and carbon fiber.

- Titanium material has high "Red/Hot Hardness". It can be worked with near-about $823 \mathrm{~K}$.

\section{References}

Ronald G. Murno, Material Properties of Titanium Diboride, National Institute of Standards and Technology. Gaitherburg, MD 20899-8520

T. Albrektsson, P. I. Branemark, H. A. Hansson \& J. Liddatrom(1981), Osseo integrated Titanium Implants: Requirements for Ensuring a Long-Lasting, Direct Bone-toImplant Anchorage in Men, Acta Orthopaedica Scandinavia, 52:2, 155-170

R. Van Noort, Titanium: The Implant Material of Today, Journal of Materials Science,(1987),3801-3811.

R. B. Subramanyam, Some Recent Innovations in The Kroll Process of Titanium Sponge Production, Bull. Mater. Sci. Vol. 16.No.6. December 1993,pp.433-451.

O.M.Ivasishin,P.E.Markovsky.Yu,V.Matviychuk,S.L.Semiatin,C. H.Ward,S.Fox, A comparative study of the mechanical properties of high-strength B-titanium alloys, Journal of alloys and compounds 457(2008)296-309.

R.R.Boyer, Titanium for aerospace: Rationale and applications, Advanced Performance Materials, October 1995,Volume 2,Issue 4,pp349-368.

N. Clement, A.Lenain, P.J.Jacques, Mechanical property optimization via microstructural control of new metastable beta titanium alloys, JOM, January 2007,Volume 59,Issue 1,pp50-53.

Tsongi Chai, Chia Kai Chou, Mechanical properties of laserwelded cast titanium joints under different conditions, The journal of prosthetic dentistry, April 1998 Volume 79,Issue 4, pp 477-483. 